\title{
Reliability and validity of the KIDSCREEN-52 questionnaire to measure health related quality of life in the 8 to 18 year-old Argentinean population
}

\author{
Silvina Berra ${ }^{a, b}$, M.D., Cristian Tebéc,d, Mg., María Eugenia Esandie.f M.D., \\ and Carlos Carignano, M.D.
}

\begin{abstract}
Introduction. The KIDSCREEN questionnaire is an instrument to measure health-related quality of life (HRQoL). It allows to differentiate health levels or to assess the efficacy of interventions. The objective was to evaluate the reliability and validity of the Argentine version of the questionnaire in the population aged 8-18 years-old in the city of Bahía Blanca.

Population and Methods. Cross sectional study conducted in public and private schools in 2008 using a district-stratified sampling. The questionnaire was self-administered and included the KIDSCREEN questionnaire (52 items) and questions about age, gender and family socioeconomic level (SEL). Reliability was analyzed using standard psychometric analysis techniques and the item response theory. The analysis of the factorial structure was performed using a confirmatory factor analysis (CFA), and that of the construct validity was conducted comparing dimension mean scores by age, gender and SEL. Results. Most dimensions had a Cronbach's alpha of $>0.7$ and $80 \%$ of items, and sufficient infit or outfit values $(<0.8)$. The CFA showed an adequate data adjustment to the ten-dimension structure. The mean values of the dimensions confirmed lower scores in adolescents than in children, and lower scores in females than in males, as expected in several of the HRQoL dimensions.
\end{abstract}

a. Consejo Nacional de Investigaciones Científicas y Técnicas (CONICET).

b. School of Public Health. School of Medicine, Universidad Nacional de Córdoba.

c. Agència d'Informació, Avaluació i Qualitat en Salut (AIAQS). Barcelona, España.

d. CIBER de Epidemiología y Salud Pública (CIBERESP).

e. Epidemiological Research Institute, Academia Nacional de Medicina de Buenos Aires.

f. Area Epidemiological Analysis of the Determinants of Health. School of Medicine. Department of Economy, Universidad Nacional del Sur, Bahía Blanca.

g. Environmental Epidemiology. Municipalidad de Bahía Blanca.

E-mail:

Silvina Berra, M.D.: silvinaberra@gmail.com

Conflict of interest: None.

Received: 02-22-2012 Accepted: 08-22-2012
Conclusions. The reliability and validity of the Argentinean version of the KIDSCREEN-52 questionnaire were acceptable; therefore its use can be recommended for the 8-18 year-old Argentinean population.

Key words: child health, adolescents, quality of life, questionnaires, test validity.

http:/ /dx.doi.org/10.5546/aap.2013.29

\section{INTRODUCTION}

The concept of health-related quality of life (HRQoL) refers to a subjective perception of the ability to perform those activities relevant to the individual, influenced by his/her health condition. Initially, such concepts referred to the physical, psychological and social dimensions of health. ${ }^{1}$ Over time, however, its multidimensions extended to wellness or function in other environments. Hence, HRQoL related statements may reflect the effects of health-disease processes or factors that influence health, and thus allow to have a common measure to assess people with different health issues or disabilities, and may be indicative of treatment efficacy. ${ }^{2,3}$

An essential attribute of HRQoL measures is that they take the individual's perspective into account. At present, there are tools that help to obtain direct answers from children and adolescents regarding relevant and specific aspects of their life, such as relations with family and friends, that children themselves point out as the most important aspect of their lives. ${ }^{4}$

Now, there are more than a hundred HRQoL questionnaires for children and adolescents, and in most of them several aspects of their validity and reliability have been assessed. ${ }^{5}$ As a result, they can be used for research, in clinical practice or in any other type of health interventions. There are selfadministered versions to be used from 4 years old on and versions for indirect informers that comprise the 0 to 18 year old range. ${ }^{5}$

Most of these instruments were developed in North America or Europe and, the most relevant Spanish language versions are those localized to Spanish populations. ${ }^{6}$

In Latin America, ${ }^{7}$ and especially in Argentina, questionnaires have been localized and some of these versions are being implemented both in clini- 
cal and public health settings for different purposes. $^{8-10}$

The KIDSCREEN ${ }^{11}$ questionnaire was created using a strict methodology in compliance with the internationally-agreed guidelines submitted by a group of experts on HRQoL. ${ }^{12,13}$ The questionnaire items were developed based on focus groups with 8 to 18 year old children and adolescents from different European countries. They were then classified in HRQoL dimensions by experts and tested in interviews with children of the same age in order to select the questions, time periods and answer scales best understood by them. ${ }^{4}$ The items were finally selected based on an analysis of the classic psychometric theory and the item response theory, so that the definitive version of the questionnaire would comply with the expected metric properties and would not be biased by age, gender or country. ${ }^{14} \mathrm{~A}$ transcultural adaptation ${ }^{15}$ was performed in Argentina so as to obtain an instrument that was equivalent to the European original version. The method included a direct and back translation of the questionnaire which involved professional linguists, cognitive interviews with people of different age, gender and socioeconomic level in order to analyze its cultural adequacy and to check the semantic equivalence between the Argentinean and the original versions. ${ }^{10}$ The objective of this article was to evaluate the reliability and validity of the Argentinean version of the KIDSCREEN questionnaire in a representative sample of 8 to 18 year old schooled population in the city of Bahía Blanca.

\section{METHODS}

Cross-sectional study conducted in public and private schools in the city of Bahía Blanca during
2008. The eligible population consisted of 8 to 18 year old male and female children and adolescents attending such facilities. Data corresponding to school enrolment provided by the General Schools Department were used to design the sample $(\mathrm{n}=51,487)$.

The sample was selected using a two-stage cluster sampling. Sampling units for the first stage were the schools stratified by district, and for the second stage, classes within each selected school. The sample size was estimated by means of a $95 \%$ confidence interval and an estimated maximum error for the parameters of $0.02(2 \%)$. A $20 \%$ nonresponse rate and an a 3 deff error (differences in variance with respect to a simple random sampling) attributable to the complex design were estimated, obtaining the necessary number of cases of 3920 students. The elemental unit was the child or adolescent selected from the sampling. Only those students whose parents had given their consent and authorized their participation in the study were included.

We used the Argentinean version of the KIDSCREEN-52 questionnaire which consists of 10 dimensions collected in 52 items (Table 1), adapted for children and adolescents aged 8 to 18 years old.

The questionnaire was self-administered. Answers to items were categorized in a 5-option Likert-type scale which assesses the frequency or intensity of the attribute over a 1-week recall period in most questions. Based on the answers, mean scores were estimated for each dimension, which were standardized to a mean of 50 and a standard deviation of 10 , according to the recommendations made by the group who developed the original version. The questionnaire also

TABLE 1. KIDSCREEN dimensions, aspects and number of items included in each of them

- Physical well-being: physical activity, feeling of energy and physical wellness (5 items).

- Psychological well-being: positive emotions and satisfaction with life (6 items).

- Moods and emotions: negative experiences, depressive moods and stressful feelings (7 items).

- Self-perception: perception of body appearance and image, and satisfaction related to them (5 items).

- Autonomy: opportunities perceived to perform activities during free time (5 items).

- Parent relations and family life: family atmosphere (6 items).

- Social support and peers: relation with peers (6 items).

- School setting: perceptions about their own cognitive capacity and concentration, and their feelings at school (6 items).

- Social acceptance/bullying: feeling rejected by peers in school (3 items).

- Financial resources: perception of the family's financial capacity (3 items). 
included age, gender and socioeconomic level (SEL) of each participant's family. The SEL was determined based on the Family Affluence Scale (FAS), an index generated according to the number of motor vehicles and computers owned by the family, if the participant has his/her own room, and having had family vacations over the last 12 months. ${ }^{16}$ The scale is made up of 7 categories (from 0 , the lowest SEL, to 7 , the highest SEL) and it is re-coded in 3 groups: low (0-3), middle (4-5) and high (6-7) SEL. ${ }^{17}$

Four people trained to conduct the pilot test and the field work introduced the study in each classroom and solved students' queries while they answered the survey.

Items internal consistency in relation to their corresponding dimension was analyzed using Cronbach's alpha coefficient, ${ }^{18}$ for which a minimum value of 0.7 was established as acceptable. Missing values and floor and ceiling effects, represented by the percentage of individuals having minimum and maximum scores, respectively, for all possible values in each scale were analyzed, up to $15 \%$ was considered as an acceptable value for three indicators. ${ }^{19}$

Based on the item-response theory and the history of original versions, unidimensionality was expected to be proven for each item and their local independence. The property of unidimensionality is accomplished when each item is accountable for a single trait or skill, while local independence is met when the likelihood of answering an item in a positive manner does not influence the answer to a different item in people with the same trait level. ${ }^{20}$ These properties were analyzed following a theoretical partial credit model (PCM), which is related to Rasch models. For the evaluation of item behavior in these models, infit and outfit statistics was used, and an item was considered adequate when the infit/outfit statistics was $0.8-1.2 .^{21}$

Finally, a confirmatory factorial analysis allowed to assess data adjustment to a pre-established structure between the questionnaire's items and their dimensions. Goodness-of-fit in data was measured using comparative fit indexes (CFIs, minimum criterion: $\geq 0.9$ ), a normed fit index (NFI, minimum criterion: $\geq 0.9$ ), and a root mean square error of approximation (RMSEA, minimum criterion: $\leq 0.06){ }^{22}$

In order to evaluate the construct validity, mean scores of the KIDSCREEN-52 dimensions were compared by age, gender and FAS scale, and the effect size was estimated calculating stan- dardized mean differences (effect size). It was established that differences of 0.2-0.5 would be minimum, those of $0.51-0.8$ would be moderate, and those higher than 0.8 would be significant. ${ }^{23}$ Statistical analyses were done with the SPSS software version 15, while the Rasch model and the confirmatory factorial analysis were done with the R software version 2.12.2.

\section{RESULTS}

Of 5236 initially selected students, $85.7 \%$ (n $=4488$ ) completed the survey. The main reasons for not answering the questionnaire were absence on the day the questionnaire was delivered $(\mathrm{n}=$ 509) and lack of parental consent to participate (n $=227$ ). Eighty six cases were excluded because students were younger than 8 years old or older than 18 years old. The sample eventually consisted of 4402 students. Of them, $43.6 \%$ were $8-12$ years old, while $56.4 \%$ were $13-18$ years old, with a similar male/female ratio. A total of $35.1 \%$ of the sample was classified as low SEL, $44.4 \%$ as middle SEL, and $20.5 \%$ as high SEL (Table 2 ).

The number of missing cases due to lack of answer to the items in one scale did not reach 5\% in any of the scales (Table 3). The floor and ceiling effect was minimum in all dimensions, except for the social acceptance dimension, with a ceiling effect reaching $31.4 \%$. Most dimensions had an internal consistency of over 0.7 , except for selfperception (alpha $=0.68)$ and social acceptance (alpha $=0.64)$.

Unidimensionality and local independence were confirmed in 37 out of 52 items. Figure 1 is an example of the results obtained in one item of the physical well-being dimension. Items with infit or outfit values lower than 0.8 specially affected financial resources and social rejection dimensions, with 2 and 3 items having this limitation, respectively. Figure 2 shows a person-item map of the same dimension. Data regarding the rest of the items and dimensions are available for readers.

The results of the confirmatory factorial analysis showed an adequate adjustment of data to the ten-dimension structure. Statistics related to the model goodness-of-fit had acceptable results (NFI $=0.89 ; \mathrm{CFI}=0.90$; RMSEA index $=0.042$ ).

Lastly, mean KIDSCREEN scores confirmed the expected differences according to age and gender. Adolescent boys and girls had lower scores in most dimensions, with an important size effect (SE) in the physical well-being (SE $=0.8)$, psychological well-being $(\mathrm{SE}=0.8)$, and 
school setting ( $\mathrm{SE}=1.1$ ) dimensions, while the social support and relation with peers dimensions were lower in children younger than 12 years old, but with minimum size effects (Table 4). In relation to mean scores per gender (Table 4), boys had higher scores than girls in four dimensions, with minimum size effects (SE $=0.2-0.4$ ), but girls had higher average scores in the school setting dimension $(\mathrm{SE}=-0.2)$.

\section{DISCUSSION}

There are still few questionnaires in Argentina available for the assessment of HRQoL in children and adolescents. ${ }^{7}$ This article shows that the reliability and validity of the KIDSCREEN questionnaire localized for the Argentinean population are adequate, and it constitutes a solid scientific base that supports its local implementation and results in a fundamental instrument for the com-

TABLE 2. Gender, socioeconomic level and parent's education level rates per age group and for the entire sample

\begin{tabular}{|c|c|c|c|c|c|c|}
\hline & \multicolumn{2}{|c|}{ Children } & \multicolumn{2}{|c|}{ Adolescents } & \multicolumn{2}{|c|}{ Entire sample } \\
\hline & $\mathbf{n}$ & $\%$ & $\mathbf{n}$ & $\%$ & $\mathbf{n}$ & $\%$ \\
\hline \multicolumn{7}{|l|}{ Gender } \\
\hline Female & 1024 & 53.3 & 1335 & 53.8 & 2359 & 53.6 \\
\hline Male & 897 & 46.7 & 1145 & 46.2 & 2042 & 46.4 \\
\hline Total & 1921 & 100.0 & 2480 & 100.0 & 4401 & 100.0 \\
\hline \multicolumn{7}{|l|}{ Socioeconomic level ${ }^{*}$} \\
\hline Low & 561 & 30.0 & 950 & 39.0 & 1511 & 35.1 \\
\hline Middle & 931 & 49.8 & 983 & 40.4 & 1914 & 44.4 \\
\hline High & 378 & 20.2 & 503 & 20.6 & 881 & 20.5 \\
\hline Total & 1870 & 100.0 & 2436 & 100.0 & 4306 & 100.0 \\
\hline \multicolumn{7}{|l|}{ Mother's education level ${ }^{*}$} \\
\hline Did not attend school & 77 & 4.3 & 39 & 1.6 & 116 & 2.8 \\
\hline Primary school & 348 & 19.6 & 507 & 20.8 & 855 & 20.3 \\
\hline Secondary school & 667 & 37.5 & 1078 & 44.2 & 1745 & 41.4 \\
\hline Tertiary school or university & 687 & 38.6 & 813 & 33.4 & 1500 & 35.6 \\
\hline Total & 1779 & 100.0 & 2437 & 100.0 & 4216 & 100.0 \\
\hline \multicolumn{7}{|l|}{ Father's education level ${ }^{*}$} \\
\hline Did not attend school & 91 & 5.3 & 53 & 2.2 & 144 & 3.5 \\
\hline Primary school & 322 & 18.8 & 584 & 24.5 & 906 & 22.1 \\
\hline Secondary school & 644 & 37.6 & 983 & 41.3 & 1627 & 39.8 \\
\hline Tertiary school or university & 655 & 38.3 & 759 & 31.9 & 1414 & 34.6 \\
\hline Total & 1712 & 100.0 & 2379 & 100.0 & 4091 & 100.0 \\
\hline
\end{tabular}

${ }^{*} P$ value of $<0.05$ in the chi-square test $(\chi 2)$ for the rate differences among age groups.

TABLE 3. Metric properties of the KIDSCREEN-52 questionnaire Argentinean version

\begin{tabular}{lcccccccc}
\hline & & & & & & \multicolumn{2}{c}{ Rash analysis: PCM } \\
\cline { 6 - 8 } & $\begin{array}{c}\text { Number of } \\
\text { items }\end{array}$ & $\begin{array}{c}\text { Missing } \\
\text { values (\%) }\end{array}$ & $\begin{array}{c}\text { Floor } \\
\text { effect (\%) }\end{array}$ & $\begin{array}{c}\text { Ceiling } \\
\text { effect (\%) }\end{array}$ & $\begin{array}{c}\text { Cronbach's } \\
\text { alpha }\end{array}$ & $\begin{array}{c}\text { Infit } \\
\text { (min-max) }\end{array}$ & $\begin{array}{c}\text { Outfit } \\
\text { (min-max) }\end{array}$ \\
\hline Physical well-being & 5 & 4.5 & 0.1 & 0 & 0.77 & $0.68-1.01$ & $0.69-1.00$ \\
Emotional well-being & 6 & 2.0 & 0.05 & 12.3 & 0.85 & $0.79-1.03$ & $0.78-1.04$ \\
Mood & 7 & 4.2 & 0.3 & 6.1 & 0.84 & $0.72-1.12$ & $0.70-1.13$ \\
Self-perception & 5 & 3.3 & 0.2 & 16.5 & 0.82 & $0.78-1.09$ & $0.62-1.07$ \\
Autonomy & 5 & 2.5 & 0.3 & 18.9 & 0.88 & $0.73-0.99$ & $0.69-0.97$ \\
Parent relations and family life & 6 & 3.4 & 0.05 & 15.4 & 0.68 & $0.74-0.89$ & $0.72-0.93$ \\
Social support and peers & 6 & 4.5 & 0.5 & 13.5 & 0.81 & $0.76-0.92$ & $0.76-0.93$ \\
School setting & 6 & 3.4 & 0.2 & 7.4 & 0.86 & $0.76-0.95$ & $0.74-0.98$ \\
Social acceptance/bullying & 3 & 2.6 & 0.6 & 31.4 & 0.65 & $0.66-0.78$ & $0.59-0.73$ \\
Financial resources & 3 & 2.7 & 2.1 & 13.1 & 0.83 & $0.64-0.82$ & $0.65-0.81$ \\
\hline
\end{tabular}

* Percentage of cases with no dimension estimated due to lack of answer in over $50 \%$ of items. 
FIGURE 1. One item characteristic curve in the physical well-being dimension

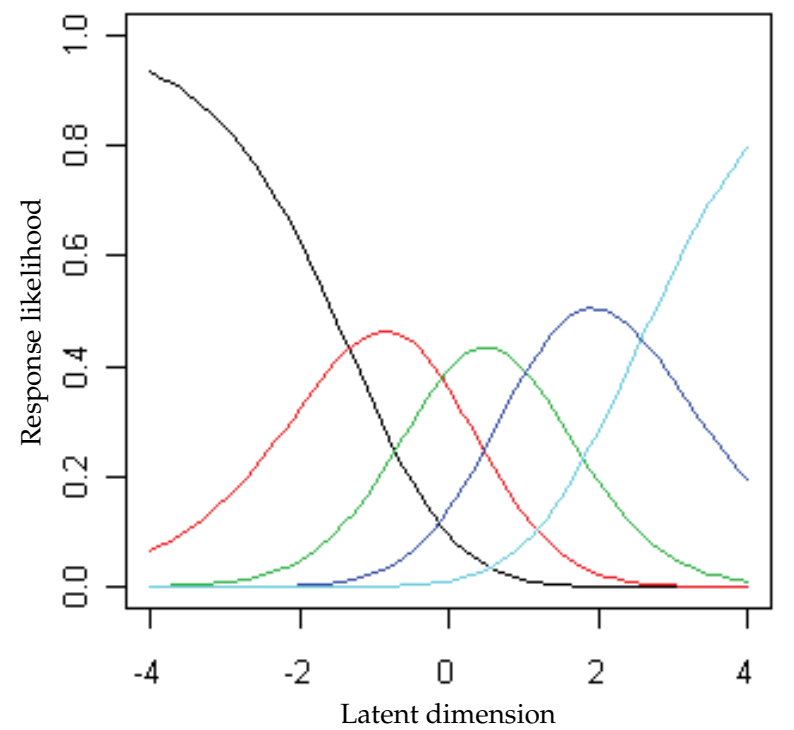

The $\mathrm{X}$ axis indicates the latent dimension value (e.g., physical well-being); the $Y$ axis represents the response likelihood. Each curve represents the distribution of individuals' response likelihood values for each analyzed item category (in this case: "Did you feel well and physically fit?"). The first curve to the left represents the lowest category ("not at all") and shows value distribution, mainly in the lower part of the latent dimension (values $<0$ ). The last curve to the right represents the highest category ("a lot") and shows value distribution, mainly in the upper part of the latent dimension (values $>0$ ). The rest of the curves represent intermediate categories and their expected values (high part of the curve), and they follow the order established by the adjacent response category.

FIGURE 2. Person-item map for the physical well-being dimension

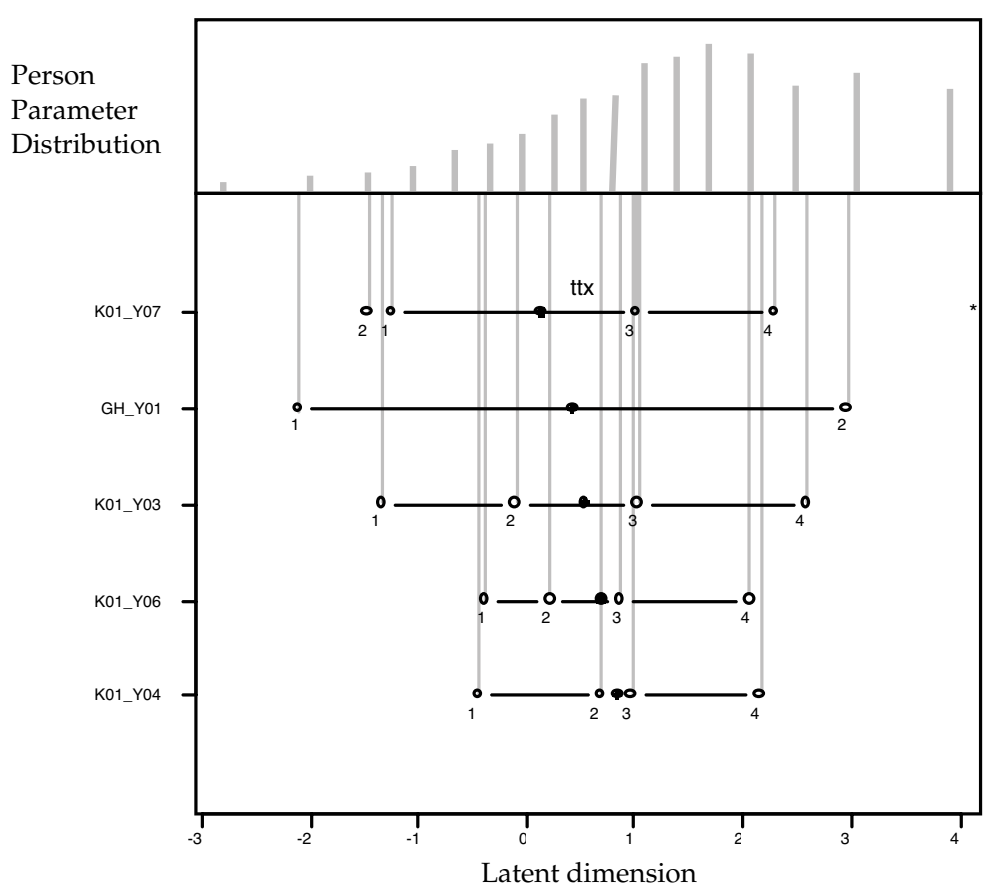

In the top of the figure, the $\mathrm{X}$ axis represents the latent dimension values (physical well-being), and the $\mathrm{Y}$ axis shows the number of boys and girls. Columns represent the number of boys and girls with a specific score in the latent dimension. The ideal situation for this dimension is a bell-shaped curve centered in high median values, with a long tail for low values (towards the left). The bottom of the figure has the same $\mathrm{X}$ axis and no $\mathrm{Y}$ axis. The points represent the answer category for one item. Each dimension item is presented horizontally. The value of each dot in the $X$ axis shows where in the latent dimension score values is each of the answer categories for each item. For a better explanatory capacity by dimension items, the ideal situation is to have a wide range of values in the latent score. 
parison of results in a regional and international context. Likewise, these results help reducing the knowledge gap regarding metric properties of instruments for their use in Spanish-speaking populations.

Internal consistency and adjustment of items that are part of the dimensions of HRQoL measurement to the Rash model had highly acceptable values in most dimensions. These results confirm that each item of the adapted version provides adequate information on the different traits of the HRQoL for children or adolescents and that each dimension offers a perceived health value under a multi-dimensional assumption.

Some reliability coefficients were somewhat lower, but within desirable levels than those obtained with the original versions, ${ }^{14,24}$ and this might be considered an excellent result for an instrument developed for a different population that after a strict cultural localization process also proves to be reliable for the Argentinean population. Therefore, results show that the measurements of the Argentinean and the original European versions are equivalent.

Similarly to what has been observed with the original KIDSCREEN version and other instruments that measure HRQoL, the construct validity analysis indicated that the KIDSCREEN version localized to the Argentinean population distinguishes the differential aspects of health by age and gender, as expected in this transitional period from childhood to adulthood with so many biological and psychosocial changes. Girls indicated a self-perception and physical well-being somewhat worse than boys, but they obtained better scores in a few of the social dimensions, such as school setting. It has also been confirmed that male and female children state a better HRQoL than male and female adolescents, except in the social acceptance dimension. Physical well-being, emotional well-being and school setting are the dimensions with the lowest scores among male and female adolescents. These HRQoL results by age and gender are consistent with those observed in the implementation of the KIDSCREEN original version in other children and adolescent populations.

One of the study limitations is that it was done in only one city of Argentina. However, in a study conducted to analyze the metric properties of a measure, significance does not rely on representativity, but on the variability provided by cases, so that it is possible to obtain wide score ranges, i.e., the sample should consist of individuals capable of scoring the entire scale of studied dimension values. The sample of this study was random and included male and female children and adolescents from public and private schools of one city of Argentina. Nonetheless, this study does not provide information on how the questionnaire would result in a non-schooled population.

Other studies should provide more evidence on the reliability and validity of the Argentinean version of the KIDSCREEN questionnaire, since there are several metric properties expected for this kind of measurement, such as the test-retest stability of the measure or the convergent and discriminant validity with similar instruments, or other measures, such as mental health or disability, and its sensitivity to changes in order to detect treatment effects or preventive interventions. This instrument is useful for different purposes, such as establishing children and adolescents health status, evaluating social or health services, both

TABLE 4. Average scores and standard deviation of the KIDSCREEN dimensions by age group and gender

\begin{tabular}{lcccccccccc}
\hline HRQoL dimensions & \multicolumn{2}{c}{ Children } & \multicolumn{2}{c}{ Adolescents } & & Males & \multicolumn{2}{c}{ Females } \\
& Mean & SD & Mean & SD & $\begin{array}{c}\text { Size effect } \\
\text { (d) }\end{array}$ & Mean & SD & Mean & SD & $\begin{array}{c}\text { Size effect } \\
\text { (d) }\end{array}$ \\
\hline Physical well-being & 53.6 & 10 & 45.6 & 9.5 & 0.8 & 51.3 & 10 & 47.1 & 10.5 & 0.4 \\
Emotional well-being & 56 & 8.9 & 48.7 & 9.6 & 0.8 & 52.4 & 9.5 & 51.4 & 10.4 & 0.1 \\
Mood & 48.3 & 10.9 & 44.2 & 9.9 & 0.4 & 47.2 & 10.3 & 44.9 & 10.6 & 0.2 \\
Self-perception & 54.1 & 10.5 & 49.4 & 9.3 & 0.5 & 52.9 & 9.6 & 50.1 & 10.3 & 0.3 \\
Autonomy & 53.1 & 10.4 & 49 & 10.3 & 0.4 & 51.8 & 10.2 & 49.8 & 10.8 & 0.2 \\
Parent relations and family life & 53.9 & 10 & 48.2 & 10.7 & 0.5 & 51.4 & 10.1 & 49.9 & 11.3 & 0.1 \\
Social support and peers & 55.9 & 10.8 & 53.9 & 10.2 & 0.2 & 54.5 & 10.3 & 54.9 & 10.6 & 0 \\
School setting & 56.9 & 11.1 & 46.2 & 8.5 & 1.1 & 49.4 & 11 & 51.9 & 11 & -0.2 \\
Social acceptance/bullying & 44.7 & 11.6 & 46 & 10.2 & -0.1 & 45.3 & 10.7 & 45.5 & 10.9 & 0 \\
Financial resources & 47.9 & 9.7 & 47.8 & 8.7 & 0 & 48.4 & 9 & 47.3 & 9.2 & 0.1 \\
\hline
\end{tabular}


therapeutic or preventive, and distinguishing the impact of different vital situations on health, using for reference the data from this sample collected in the city of Bahía Blanca, as well as the values provided by the original versions from 13 European countries in order to make comparisons.

\section{CONCLUSION}

This study shows that the reliability and validity of the Argentinean version of the KIDSCREEN-52 questionnaire are acceptable to be used in HRQoL research in the Argentinean population aged 8-18 years old.

\section{Acknowledgments}

To Silvina Spagnolo, Magister, Paula Abrego, Magister, and Lilian Elosegui, M.D. specialized in Environmental Epidemiology of the Municipality of Bahía Blanca, for their worthy help in this study's field work.

\section{BIBLIOGRAPHY}

1. Testa MA, Simonson DC. Assessment of quality of life outcomes. N Engl J Med 1996;334:835-40.

2. Patrick D, Erickson P. Health Policy, Quality of Life: Health Care Evaluation and Resource Allocation. Nueva York: Oxford University Press; 1993.

3. Békési A, Török S, Kökönyei G, Bokrétás I, Szentes A, Telepóczki $G$ and The European KIDSCREEN Group. Health-related quality of life changes of children and adolescents with chronic disease after participation in therapeutic recreation camping program. Health and Quality of Life Outcomes. 2011;9(43). [Accessed on: February 2012]. Available at: http://www.hqlo.com/content/9/1/43.

4. Detmar SB, Bruil J, Ravens-Sieberer U, Gosch A, Bisegger $C$; the European KIDSCREEN group. The Use of Focus Groups in the Development of the KIDSCREEN HRQL Questionnaire. Qual Life Res 2006;15(8):1345-53.

5. Solans M, Pane S, Estrada MD, Serra-Sutton V, Berra S, Herdman M, Alonso J, Rajmil L. Health-related quality of life measurement in children and adolescents: a systematic review of generic and disease-specific instruments. Value Health 2008;11(4):742-64.

6. Bibliopro: Biblioteca virtual de Resultados Percibidos por los Pacientes (PRO - Patient Reported Outcomes). Available at: http://bibliopro.imim.es

7. Rajmil L, Roizen M, Urzúa A, Hidalgo-Rasmussen C, et al; Working Group on HRQOLin Children in Ibero-American Countries. Health-Related Quality of Life Measurement in Children and Adolescents in Ibero-American Countries, 2000 to 2010. Value Health 2012;15(2):321-22.

8. Moroldo MB, De Cunto C, Hübscher O, Liberatore D, et al. Cross-cultural adaptation and validation of an Argentine Spanish Version of the Stanford Childhood Health Assess- ment Questionnaire. Arthritis Care Res 1998;11(5):382-90.

9. Roizen M, Rodríguez S, Bauer G, Medin G, et al. Initial validation of the Argentinean Spanish version of the PedsQL 4.0 Generic Core Scales in children and adolescents with chronic diseases: acceptability and comprehensibility in low-income settings. Health Qual Life Outcomes 2008;6:59.

10. Berra S, Bustingorry V,HenzeC, Díaz MP, et al. Adaptación transcultural del cuestionario KIDSCREEN para medir la calidad de vida relacionada con la salud en población argentina de 8 a 18 años. Arch Argent Pediatr 2009;107(4):30714.

11. The KIDSCREEN Group Europe. The KIDSCREEN Questionnaires. Quality of life questionnaires for children and adolescents. Lengerich: Pabst Science Publishers; 2006.

12. Scientific Advisory Committee of the Medical Outcomes Trust. Assessing health status and quality-of-life instruments: attributes and review criteria. Qual Life Res 2002;11:193-205.

13. Herdman M, Rajmil L, Ravens-Sieberer U, Bullinger M, Power M, Alonso J; European Kidscreen Group European Disabkids Group. Expert consensus in the development of a European health-related quality of life measure for children and adolescents: a Delphi study. Acta Paediatr 2002;91(12):1385-90.

14. Ravens-Sieberer U, Gosch A, Rajmil L, et al; KIDSCREEN Group. The KIDSCREEN-52 quality of life measure for children and adolescents: psychometric results from a crosscultural survey in 13 European countries. Value Health 2008;11(4):645-58.

15. Beaton DE, Bombardier C, Guillemin F, Ferraz MB. Guidelines for the process of cross-cultural adaptation of selfreport measures. Spine 2000;25:3186-91.

16. Currie $\mathrm{C}$, et al. Indicators of socioeconomic status for adolescents: the WHO Health Behaviour in School-aged Children survey. Health Educ Res 1997;12(3):385-97.

17. Currie CE, Elton RA, Todd J, Platt S. Indicators of socioeconomic status for adolescents: the WHO Health Behaviour in School-aged Children survey. Health Educ Res 1997;12(3):385-97.

18. Cronbach JL. Coefficient alpha and internal structure of tests. Psychometrika 1951;16:297-334.

19. Nunnally JC, Bernstein IH. Psychomertric theory. 3rd ed. Nueva York: McGraw-Hill; 1994.

20. Embretson SE, ReiseSP. Item response theory for psychologists. Londres: Lawrence Erlbaum Associates, Inc.; 2000.

21. Streiner D, Norman G. Health Measurement Scales. A practical guide to their development and use. Nueva York: Oxford University Press; 1993.

22. Byrne MB. Structural equation modeling with LISREL, PRELIS, and SIMPLIS: basic concepts, applications and programming. Londres: Lawrence Erlbaum Associates; 1993.

23. Cohen J. Statistical power analysis for the behavioral sciences. 2nd ed. Hillsdale: Lawrence Erlbaum Associates, Inc.; 1998.

24. Tebe C, Berra S, Herdman M, Aymerich M, Alonso J, Rajmil L. Fiabilidad y validez de la versión española del KIDSCREEN-52 para poblacióninfantily adolescente. Med Clin (Barc) 2008;130(17):650-4 